\title{
«Ne pas rester lié à sa propre rupture » Solitude et communauté dans la pensée de Nietzsche
}

\author{
Céline Denat
}

Pour Nietzsche, le philosophe doit avant tout être un «esprit libre », que caractérise la vertu d'indépendance, c'est-à-dire une tendance spontanée à s'affranchir de tout lien, même à l'égard de ce et de ceux qui lui sont d'abord le plus chers. Le philosophe est donc celui qui pense autrement que ne pensent la plupart de ceux qui l'entourent, et qui interroge là où nul n'a le courage ni même l'idée de poser des questions. Pour ces raisons, il se caractérise aussi par une nécessaire solitude: ses expériences et ses pensées le conduisent, du fait de leur originalité, « toujours plus loin, toujours plus à l'écart. La solitude le cerne et l'enserre, de plus en plus menaçante, étouffante, lancinante » (Humain, trop humain I, préface $\S 3)^{1}$. Voyage, exil, désert, air raréfié des hauteurs et des glaces, sont alors autant de métaphores bien connues, au travers desquelles Nietzsche affirme, tout au long de son cuvre $^{2}$, la solitude du penseur, que ses perspectives nouvelles isolent nécessairement.

Le philosophe tel que le pense Nietzsche apparaît en effet trois fois condamné à la solitude. Son refus de se contenter de penser et de parler avec le plus grand nombre doit tout d'abord nécessairement le conduire à prendre ses distances à l'égard de la foule qui pourrait sans cela l'entraîner à l'oubli de lui-même et de ses exigences propres, comme l'indique cet aphorisme d'Aurore $(\$ 491)$ : «je vais dans la solitude, — pour ne pas boire aux citernes communes. Dans la foule, je vis comme la foule et ne pense pas selon mon être; au bout d'un 
certain temps, j'ai toujours l'impression que l'on veut m'exiler de moi et me ravir mon âme [...]. Le désert m'est alors nécessaire pour redevenir bon. » Mais il faut dire en outre que cette solitude n'est pas l'effet d'un choix libre et raisonné : étant de sa nature même un homme « hors du commun », c'est de manière tout instinctive, en vertu d'un " penchant» et d'une «inclination » nécessaires, que le philosophe à l'esprit libre aspire « à sa citadelle et sa retraite secrète où il soit délivré de la foule, du grand nombre, de la majorité, où il puisse oublier la règle "homme", lui qui en est l'exception »(Par-delà bien et mal § 284 et $\S 25)$. Enfin il faut admettre que, eût-il encore le désir d'échapper à sa solitude, le philosophe authentique ne le pourrait guère : l'art du soupçon ainsi que les «différence[s] de vue » qui le caractérisent le condamnent nécessairement «[aux] glaces et [aux] angoisses de l'isolement » (Humain, trop humain I, préface $\S 2 ; c f$. Tongeren 217 sq.). S'il se détourne d'abord de la foule, celle-ci ne peut en retour que le considérer avec défiance et le rejeter, puisque l'extrême originalité de ses recherches et de ses écrits fait qu'il se heurte à l'incompréhension de ses semblables, et de ceux mêmes qui lui furent le plus proches, comme l'indique entre autres la préface d'Aurore :

celui qui marche ainsi sur ses voies propres n'y rencontre personne : cela tient à la nature de ces « voies propres ». Personne ne vient l'aider dans son entreprise : dangers, hasards, méchancetés et tempêtes, tout ce qui l'assaille, il doit le surmonter lui-même. C'est qu'il a son chemin à lui - et, bien sûr, l'amertume, le dépit que lui cause à l'occasion cet « à lui »: auxquels contribue, par exemple, le fait que ses amis eux-mêmes ne peuvent deviner ni où il est, ni où il va... ( $(2)$

Mais le rappel de ce propos, somme toute bien connu, de Nietzsche quant à l'indépendance, et en conséquence quant à la solitude du philosophe, nous confronte cependant d'emblée à une difficulté. Car il faut bien apercevoir ici un étrange paradoxe, qui consiste en ce que, tout en prétendant à une radicale «différence de vue » qui le condamnerait à l'isolement, Nietzsche semble bien reprendre néanmoins ce qui constitue certainement un lieu commun, non 
seulement à propos du philosophe, mais aussi de la part des philosophes eux-mêmes, qui ont avant lui insisté sur la nécessité d'une telle solitude pour celui qui entend goûter les joies et les rigueurs de la pensée. Que l'on songe par exemple au philosophe platonicien, qu'isole et que condamne l'ignorance de ses contemporains (cf. République VII, 517a); à la contemplation solitaire et à l'autosuffisance quasi divines du sage aristotélicien (cf. Éthique à Nicomaque X, 7, $1177 \mathrm{a} 27$ sq.); à la requête épicurienne d'une «vie tranquille et à l'écart de la foule » (Maximes fondamentales XIV); à l'exigence cartésienne d'un «repos» propice à la méditation et qu'autorise seule une «paisible solitude » (Méditations métaphysiques I); ou encore au refus pascalien du divertissement et du monde, qui conduisent à l'oubli de soi, et du caractère tragique de notre existence (Pensées, Brunschvicg 139) ${ }^{3}$ - la plupart des penseurs semblent d'accord pour affirmer la solitude inhérente à cette figure exceptionnelle qui est celle du philosophe. Et c'est d'ailleurs parfois avec l'accent ou les formules de certains de ses prédécesseurs que Nietzsche semble à son tour exprimer cette même exigence, requérant par exemple avec des accents quasi pascaliens que l'on apprenne à « supporter la solitude » (Nietzsche, Aurore § 443) et à affronter l'ennui pour «boire la plus tonique des gorgées à sa propre source intérieure » (Le voyageur et son ombre $\S 200)^{4}$; ou reprenant parfois l'image épicurienne du jardin, de la vie cachée et à l'écart, à titre de conditions de la réflexion philosophique (Gai savoir $§ 280$ et $\S 338)^{5}$. Mais on est alors en droit de se demander si la prétention nietzschéenne à une radicale indépendance ne cède pas au moins sur ce point précis, c'est-à-dire si cette exigence d'un écart et d'une distance ne le conduit pas justement à renoncer à la distance qu'il prétend creuser entre luimême et le plus grand nombre. Ce penseur qui exige que l'on ne veuille pas rejoindre le « chœur » des modes de pensée les plus communs, que l'on ne sache pas même comment «faire le chœur » (Aurore § 177), ne fait-il pas ici chorus à un lieu commun de la philosophie, et par là 
ne se contredit-il pas lui-même? Et par ailleurs : une telle prétention à la solitude peut-elle se concilier sans contradiction avec la volonté de s'adresser à et d'être entendu par d'autres, d'écrire au bénéfice d'un public, ce qui implique nécessairement le risque, comme le reconnaît Nietzsche lui-même, de se «rendre commun» (Crépuscule des idoles, «Incursions d'un inactuel » § 26 et Par-delà bien et mal § 284)?

Sans doute faut-il commencer par dire qu'une différence majeure sépare la solitude philosophique telle que la pense la tradition, de celle qu'exige Nietzsche : pour la première, le philosophe se trouve isolé de la communauté des hommes non seulement du fait des exigences inhérentes à sa recherche, mais aussi en raison des vérités qu'il découvre, et dont les autres demeurent ignorants. La «solitude du philosophe» consiste alors, comme le remarquait F. Alquié, en une paradoxale «solitude de l'universalité », qui vient de ce qu'il découvre une vérité qui devrait appartenir à tous, mais que la plupart ignorent, faute de s'être pliés aux rigueurs du questionnement philosophique. À l'inverse, dans le contexte d'une pensée qui, comme c'est le cas chez Nietzsche, récuse radicalement la possibilité d'une vérité et d'une connaissance absolues au profit d'une théorie de l'interprétation, et qui reconnaît dans le même temps son propre caractère interprétatif, la solitude du philosophe ne saurait certes plus consister en une telle «solitude de l'universalité ». Parce qu'elle met en place un questionnement radicalement nouveau, qui vise à interroger non plus la vérité, mais la valeur des interprétations ${ }^{6}$, ou la « valeur des valeurs » qui informent nos manières de penser et d'agir, la philosophie doit bien plutôt induire une solitude qui tient au caractère insolite (ungewöhnlich), à l'extrême singularité d'un questionnement qui renonce précisément à l'exigence commune de vérité et d'universalité.

Mais il faut aller plus loin encore. Car Nietzsche ne laisse pas précisément de reprocher à ses prédécesseurs de n'avoir pas suffisamment pensé la, ou plutôt les significations de «la » 
solitude, et d'être demeurés, sur ce point comme sur d'autres, des penseurs superficiels : il reste encore à élaborer, nous dit Nietzsche, une «théorie de la société et de la solitude » (Aurore $\S 453$ ), plus précisément encore une «histoire » du sentiment de solitude (§ 249), dont on a jusqu'ici ignoré le caractère complexe, et divers. Ici comme ailleurs, l'unité du mot a trop souvent conduit à croire à la simplicité et à «l'unité de la chose » (Humain, trop humain I § 14). Nietzsche entend à l'inverse montrer que la solitude ne peut être rigoureusement pensée qu'à réfléchir de manière fine le sens et le statut de l'individu, ainsi que ceux des relations qui lient, ou au contraire séparent les hommes; comme aussi les formes variées de solitudes, qui toutes ne s'articulent pas aux mêmes besoins ni aux mêmes buts, et dont le sens et la valeur ne sont alors en rien identiques. Toute solitude n'est pas un signe de force, de santé, moins encore d'indépendance. Il peut arriver qu'un homme se retire du monde par crainte de s'y confronter, prétendant à cette fin contempler une « vérité » dont il serait le seul possesseur; ou encore qu'un individu adopte le rôle héroïque du sage érémitique, dans le seul but de mieux répondre aux attentes de la foule, et pour mieux la séduire de ce fait ${ }^{7}$. Il est donc des solitudes prétendues qui ne sont que le masque de l'homme asservi aux manières les plus communes de penser. Dans cette perspective, on s'attachera ici à étudier l'enquête historique, et l'examen axiologique, que Nietzsche mène à l'égard des solitudes, et qui le conduisent pour finir à penser la solitude (Einsamkeit, Alleinsein) philosophique d'une manière originale. Celle-ci ne se confond en effet pas avec ce qu'il désigne sous le nom d'«isolement»(Vereinsamung, Isolation), ou plus rarement d'« abandon» (Verlassenheit) ${ }^{8}$, et qu'il dénonce comme un danger qui rendrait vaines la tâche et la figure même du philosophe. Car si celui-ci doit être pensé comme un «médecin de la culture », il ne saurait en effet se tenir à jamais éloigné des hommes et des valeurs qu'il prétend non seulement évaluer, mais aussi transformer. Pour cette raison, le « je » du philosophe 
solitaire apparaît chez Nietzsche indissociable d'un «nous », qui indique qu'il lui est toujours nécessaire de s'inscrire dans une communauté, soit présente, soit à venir.

\section{Solitude et multitude : le soi comme autres}

Il est manifeste qu'aux yeux de Nietzsche, une authentique solitude est tout à la fois nécessaire, et cependant on ne peut plus difficile à atteindre. «Qui donc est jamais seul! », nous dit en ce sens le titre d'un aphorisme d'Aurore (\$249), qui semble indiquer la vanité d'une telle prétention à être jamais véritablement seul. C'est qu'en effet la solitude ne tient pas seulement à la distance spatiale que l'on est susceptible de mettre entre soi et ses semblables, non plus qu'à l'absence effective de ceux-ci. Un homme d'exception peut se trouver « aussi seul dans les villes les plus populeuses que dans un désert» (Gai savoir § 2). Et il peut aussi bien arriver, à l'inverse, que celui qui s'exile loin de sa communauté n'en continue pas moins de lui appartenir, dans la mesure où il continue de vivre selon les croyances qui la caractérisent, comme s'il ne s'en était jamais éloigné. La solitude n'est pas avant tout question d'espace, de présence ou d'absence, et c'est pourquoi Nietzsche requiert que, loin d'une telle vision simplificatrice, on apprenne enfin ce qu'est la solitude ( $c f$. Aurore $§ 177$ ), afin de pouvoir éventuellement prétendre se faire authentiquement solitaire : «Peu à peu, la lumière s'est faite en moi sur le défaut le plus répandu de notre type de formation et d'éducation : personne n'apprend, personne n'aspire, personne n'enseigne - à supporter la solitude » (Aurore $§ 443)$. Mais « qui sait aujourd'hui ce que c'est, la solitude?... (Humain, trop humain I, préface $\S 3$ ).

Or, un tel apprentissage suppose d'abord que l'on repense le sens de l'individualité, ou du sujet humain, généralement conçu comme sujet substantiel unifié et autonome, comme soi qui ne serait qu'extrinsèquement lié à d'autres. Nietzsche s'oppose nettement à une telle conception, et 
en ce sens, à la notion même d'individu telle qu'elle est usuellement pensée : «Le concept d'“individu" est faux », nous dit ainsi un fragment posthume, si par «individu » on entend penser une unité in-divisible (FP XI 34 [123] $)^{9}$. Ce que révèle en effet un examen rigoureux, c'est qu'il y a en tout homme, non pas seulement une multiplicité de «facultés », qui seraient elles-mêmes des unités dernières; mais que ces prétendues «facultés »— dont Nietzsche récuse alors l'existence — recouvrent elles-mêmes une multiplicité de sentiments, d'affects, de besoins, parfois d'ailleurs obscurs et difficiles à percevoir, et au-delà desquels ne peut jamais être aperçue aucune unité substantielle dernière. Ainsi faut-il apercevoir par exemple que «la » prétendue volonté est «quelque chose de compliqué, quelque chose qui n'a d'unité que verbale » (Par-delà bien et mal § 19), et il faut reconnaître de même la complexe multiplicité qu'implique notre faculté de penser. Ce n'est aux yeux de Nietzsche qu'à des fins de simplification que l'on a voulu réduire l'homme au concept d'« individu », entendu en son sens étymologique, à la notion d'ego ou de «substance » pensante, comme le montre de façon particulièrement nette le Crépuscule des idoles (« La "raison" dans la philosophie » § 5; «Les quatre grandes erreurs » § 3). Il faut bien plutôt reconnaître que «le Moi n'est pas l'affirmation d'un être face à plusieurs (instincts, pensées, etc.) », mais qu'il est lui-même une «pluralité de forces personnalisées dont tantôt l'une et tantôt l'autre passe au premier plan en qualité d'ego » (FP Aurore 6 [70]; cf. FP XI 34 [123]). Si l'on peut continuer d'user du terme d'individu, c'est en reconnaissant enfin son caractère pluriel, complexe, et mouvant. C'est pourquoi Nietzsche repense à nouveaux frais l'homme, non comme un sujet, non plus que comme union substantielle d'une âme et d'un corps, mais comme étant « corps, et rien de plus » (Ainsi parlait Zarathoustra, « Des contempteurs du corps »), corps qui doit lui-même être pensé comme totalité psycho-physiologique multiple, comme ensemble organisé et hiérarchisé de pulsions, de forces, d'instincts ou d'affects, dont la pensée consciente 
n'est que l'aspect le plus superficiel. Ce corps qu'est l'homme doit être conçu, suivant la formule du $§ 19$ de Par-delà bien et mal, comme une «structure sociale composée de nombreuses âmes », l'«âme» pouvant être définie de son côté par le $\S 12$ du même ouvrage comme «structure sociale des pulsions et des affects », suivant une structure de renvoi qui interdit désormais de penser l'existence d'aucune unité dernière et fondamentale ${ }^{10}$. « Le » sujet tel que le repense Nietzsche se conçoit comme multiplicité et jeu de forces qui ne doivent elles-mêmes pas être pensées comme de nouveaux « atomes » ou « substances ». Bref, pour Nietzsche, l'individu «n'est unité qu'en tant qu'organisation et jeu d'ensemble: tout comme une communauté humaine est une unité, et pas autrement $\gg(\text { FP XII } 2 \text { [87] })^{11}$.

La métaphore de la «communauté » ou de la «structure sociale », dont use régulièrement Nietzsche pour dire cette multiplicité du «sujet » doit ici être prise au sérieux dans toute son ampleur. Car s'il s'agit sans doute de penser d'abord le caractère complexe mais organisé de cet ensemble de pulsions qu'est l'individu humain, à l'aide d'une analogie politique somme toute classique, il s'agit cependant aussi de penser la présence implicite de l'altérité, de la communauté, plus précisément des valeurs qui caractérisent une culture, au sein des individus eux-mêmes. Il faut dire en effet que les «instincts» ou «pulsions » qu'évoque Nietzsche ne doivent en rien être pensés comme des principes naturels et immédiats. Loin de tout naturalisme et tout fixisme, Nietzsche pense au contraire les instincts sur un mode toujours relationnel et processuel. Nul instinct n'a de signification ou de valeur en lui-même, mais toujours dans le contexte des relations qu'il entretient avec d'autres qu'il domine ou auxquels — tour à tour — il se subordonne et obéit: on n'a pas affaire ici à une conjonction d'unités seulement extrinsèquement liées, mais à des instances qui n'existent que dans et par le réseau de relations qui les lient. Nietzsche note en ce sens que pour penser l'homme comme ensemble de pulsions, 
« une simple addition ne suffit absolument pas. Notre arithmétique est une chose trop grossière pour ce genre de relations; elle n'est qu'une arithmétique des cas isolés [eine EinzelArithmetik] » (FP XI 34 [123]). Par ailleurs, les relations entre pulsions ne sont pas fixes, mais changeantes, et c'est pourquoi aussi les pulsions ont en quelque sorte une histoire, et peuvent se transformer, s'affaiblir, se développer, ou bien encore s'affiner ou se « sublimer $^{12}{ } »$ au point de paraître changer de nature. L'instinct de cruauté, par exemple, qui aux premiers temps de l'histoire humaine s'est souvent déchargé de manière brutale, s'est trouvé modifié progressivement par le développement d'autres besoins, en particulier sous la pression du besoin de communiquer avec d'autres et d'être soutenu par eux. Ce même instinct de cruauté n'en continue pas moins de jouer un rôle, y compris au sein des sentiments que nous qualifions comme «moraux», par exemple lorsque nous éprouvons de la bienveillance ou de la compassion à l'égard d'autrui. Car il faut voir selon Nietzsche que de tels sentiments sont encore une manière de manifester notre force à l'égard de ceux qui nous apparaissent plus faibles, et de nous les approprier en exerçant à leur égard tel ou tel acte charitable (Par-delà bien et mal § 224; FP Gai savoir 11 [124]). La cruauté s'est ici sublimée, spiritualisée du fait de la concurrence d'autres instincts en chaque individu, et dans le même temps aussi — indissolublement — du fait des relations existant entre individus, et des besoins de chacun d'eux.

Ces jeux de relations pulsionnelles, intra-individuelles et, dans le même temps, interindividuelles, permettent de comprendre pourquoi l'organisation pulsionnelle propre à un certain type d'homme, c'est-à-dire aussi les besoins spécifiques qui dominent en lui, ne peuvent être pensés indépendamment des relations naturelles ou sociales qui le font se rencontrer avec d'autres. Dans le cas de l'homme, que caractérise un mode de vie communautaire depuis un 
temps immémorial, et qui a été transformé sur une longue durée par un tel mode de vie, il faut dire que

[1]'égoïsme naïf de l'animal est totalement altéré par notre entraînement social [...]. Les tendances sociales (comme l'hostilité l'envie la haine) (qui présupposent une pluralité) nous ont métamorphosés : nous avons transposé en réduction «la société » en nous, et le fait de nous replier sur nous-mêmes ne représente pas une fuite hors de la société, mais très souvent une pénible continuation en rêve et une interprétation de nos processus internes selon le schéma de nos expériences précédentes. (FP Aurore 6 [80]; cf. Aurore $\S 105)$

Les relations entre processus pulsionnels ne sont pas seulement à penser par analogie avec les relations sociales; elles sont intrinsèquement conditionnées par les «tendances sociales » qui règlent les relations entre individus (entre complexes pulsionnels). Les valeurs, c'est-à-dire les préférences fondamentales inconscientes qui régissent une communauté, règlent non seulement les relations inter-individuelles, mais aussi les relations inter-pulsionnelles. Ainsi par exemple, dans une culture qui, comme c'est le cas de l'Europe moderne, refuse toute idée de cruauté et d'inégalité, pour valoriser absolument le respect et l'amour du prochain, l'individu répugnera souvent spontanément à toute forme explicite de cruauté et par conséquent de domination — y compris en et à l'égard de lui-même, ce pourquoi il se caractérisera par une «anarchie des instincts » qui fera généralement obstacle à l'accroissement de ses forces propres (cf. Par-delà bien et mal $\S 258$ et $\S 202$ ). Au contraire, dans le contexte de cultures (que Nietzsche caractérise comme «aristocratiques ») qui non seulement ne condamnent pas, mais valorisent la capacité de commander, d'exercer sa force au sein de relations de lutte et de domination, la tendance à imposer un ordre hiérarchique se réalisera non seulement d'individu à individu, mais aussi au sein des individus eux-mêmes, comme l'indique le $\$ 257$ de Par-delà bien et mal : la capacité d'imposer des distances d'homme à homme, de vivre dans une communauté que caractérise avant tout « la différence incarnée des positions », conduit également pour finir «à un incessant 
accroissement de distance au sein de l'âme elle-même » et par là à l'élaboration d'états «toujours plus élevés, plus rares, plus lointains ». La relation d'un individu à d'autres et à sa communauté n'est donc pas une relation simplement extrinsèque. L'individu ne peut être pensé comme un «atome » juxtaposé à d'autres, dont il lui suffirait de s'écarter pour s'en séparer. La remise en cause de la «fausse substantialisation du Moi » doit également conduire à rejeter la «fausse autonomisation de 1'“individu" en tant qu'atome» (FP XIII 10 [57]). S'il peut être pensé comme le «fruit de la vie commune » (FP IX 7 [258]), ce n'est pas suivant le schème d'une causalité mécanique externe : les modes relationnels induits par les valeurs caractéristiques d'une culture particulière sont assimilés ou incorporés par l'individu, de telle sorte que l'organisation et la hiérarchie pulsionnelles internes qui le caractérisent sont pensés comme indissociables des relations sociales externes.

Il faut encore rappeler à cet égard que la pensée consciente, que l'on tend à considérer comme constitutive de l'essence de l'homme, mais qui n'est pour Nietzsche que «sa partie la plus superficielle », puisqu'elle n'est qu'un épiphénomène du corps conçu comme ensemble de pulsions, doit elle-même être comprise comme n'étant «proprement qu'un réseau de relations d'homme à homme». Elle ne s'est en effet «développée que sous la pression du besoin de communication », du besoin de signifier à d'autres ses besoins propres et d'obtenir leur soutien; «l'homme érémitique et prédateur, ajoute Nietzsche, n'aurait pas eu besoin d'elle » (Gai savoir $\S 354)$. Le développement de la conscience n'est que le corrélat de celui de la communication entre hommes, c'est-à-dire d'un processus visant à se rendre communicable, et par conséquent commun. L'usuelle survalorisation de la conscience conduit alors l'individu à négliger ce qu'il y a peut-être encore en lui de singulier, au profit de cela seul qui peut être compris par d'autres, de sorte qu'il se réduit pour finir à «ce qui en lui est nature communautaire et grégaire ». Cela 
confirme nettement que la relation d'un individu à ses semblables n'est ni extrinsèque, ni univoque. Un individu ne vit pas seulement au sein d'une communauté. Il est tout entier habité par celle-ci, dans la mesure où son «intériorité » même se trouve être le résultat de son statut d'«animal social » (\$ 354); et puisque ses manières prétendument «propres » de penser se trouvent profondément structurées par un langage commun, aussi bien que par les valeurs inhérentes à la culture qui est la sienne.

On voit que Nietzsche repense les concepts de relation et d'altérité comme impliquant un nécessaire processus d'intériorisation de la part de tout individu. Une telle intériorisation, que Nietzsche désigne également par les termes d'assimilation et d'incorporation, ne s'effectue pas seulement à l'échelle de l'existence individuelle : elle est pensée comme le résultat d'un long devenir, d'une longue histoire ${ }^{13}$, plus spécifiquement encore d'une longue hérédité ${ }^{14}$ qui suppose que ces valeurs ont été lentement assimilées au long des générations, au point d'exercer, sur tous ceux dont le corps en est désormais le « dépositaire » (Par-delà bien et mal § 200), une autorité absolue — autorité d'autant plus incontestable que ces préférences deviennent et demeurent pour finir inconscientes ${ }^{15}$. L’individu apparaît ainsi comme étant davantage et autre que « luimême » : « il est toute la ligne homme dans son unité jusqu'à lui-même inclus » (Crépuscule des idoles, «Incursions d'un inactuel » § 33). C'est pourquoi les hommes ne peuvent être d'emblée pensés comme des personnes, des individus singuliers : «certains d'entre eux sont plusieurs personnes, la plupart n'en sont aucune » (FP XIII 10 [59]).

Or, cela conduit nécessairement Nietzsche à repenser et à déplacer le problème de la solitude. L'altérité, l'être ou le vivre-avec, les normes communes, plus généralement les conditions de l'aliénation individuelle, ne se situent pas seulement hors de nous; elles sont aussi et même surtout en nous, et c'est pourquoi nul éloignement spatial ne peut suffire à nous en 
déprendre. Comment alors prétendre sans vanité à une solitude véritable, puisqu'il semble désormais que nous ne saurions échapper à la multitude sans nous perdre également nousmêmes? Une simple fuite loin du «tumulte et du contact direct» avec ses semblables et son époque ne peut être qu'illusoire, puisque la foule vit en nous, bien plus que nous ne vivons en elle. L'«atmosphère» caractéristique de l'époque présente, qui résulte d'un long passé, «s'insinue en nous continuellement par mille veines, à chaque respiration », de telle sorte qu'il semble qu'il n'y ait «pas de solitude qui soit assez solitaire et lointaine » pour permettre de lui échapper (Sur l'avenir de nos établissements d'enseignement IV, 145). C'est pourquoi il convient de dépasser les trop simples alternatives entre soi-même et l'autre, entre individu et communauté, entre solitude et multitude, qui sont en dernière analyse superficielles et insuffisantes, pour penser à nouveaux frais la possibilité d'une solitude authentique.

\section{Pour une « histoire de ce sentiment subtil que l'on nomme solitude »}

C'est dans ce contexte qu'il faut comprendre le sens précis de cette exclamation : «Qui donc est jamais seul! », que nous avons évoquée plus haut. Cette formule n'est désespérée qu'en apparence, puisque l'aphorisme qui lui fait suite a surtout en vue de mettre en évidence ce caractère problématique, jamais suffisamment affronté, de la solitude : «Qui donc est jamais seul! — L'homme craintif ignore ce que c'est que d'être seul : il y a toujours un ennemi derrière sa chaise. - Oh, qui pourrait nous conter l'histoire de ce sentiment subtil que l'on nomme solitude! » (Aurore § 249) L'évocation de l'«homme craintif » constitue bien plus ici qu'un simple exemple parmi d'autres. Il faut rappeler en effet que la crainte constitue aux yeux de Nietzsche l'un des affects dominants au sein de l'homme tel qu'il a généralement existé jusqu'ici : les moyens variés que l'on a successivement inventés au cours de l'histoire pour 
apaiser « le sentiment d'impuissance et de peur », écrit-il, « constituent presque une histoire de la culture » (§ 230). C'est la peur qui a jusqu'ici conduit les hommes au besoin de vénérer et de se soumettre à une autorité absolue - celle des coutumes, celle de puissances suprêmes, ou bien encore, dans le cas des philosophes, celle de l'idée de vérité ou de bien absolus, qui sont autant de moyens susceptibles de mettre fin à tout questionnement et à toute inquiétude. Ainsi, lorsque Nietzsche écrit que «l'homme craintif ignore ce que c'est que d'être seul», ce n'est pas seulement pour dénoncer l'irrationalité de l'homme pusillanime, qui est conduit à imaginer partout, à tort, la présence d'un ennemi caché. Ce qu'il faut comprendre d'abord, c'est que pour l'«homme craintif », se sentir perpétuellement entouré d'ennemis constitue encore un moyen paradoxal de répondre au besoin de ne pas se sentir abandonné à lui-même. Mais s'il est vrai plus généralement que l'instinct de peur joue à différents degrés en tout homme, il faut comprendre également qu'il s'accompagne généralement d'un tel besoin de n'être pas délaissé, et par suite de se rapporter à une altérité, le cas échéant à une autorité supérieure. Cette autorité, et les jugements et condamnations qu'elle rend possibles, sont toujours déjà là, en tout individu, ou pour ainsi dire «derrière sa chaise », prêts à le punir s'il tentait de s'y soustraire. Ce qui revient à dire que l'homme «seul dans une chambre », mais qui continue d'être soumis à l'affect de crainte et, en conséquence, à un besoin de vénération et de soumission inaperçu, ne peut en aucun cas être authentiquement solitaire.

Mais «l'histoire de ce sentiment subtil que l'on nomme solitude» se trouve encore approfondie par Nietzsche. Celui-ci montre en effet plus précisément que l'époque moderne se caractérise par la soumission aux valeurs issues du platonisme et du christianisme, c'est-à-dire par des idéaux «grégaires » et «démocratiques » valorisant l'idée de bien commun et d'égalité. Elle se caractérise également, en conséquence, par la survalorisation de l'altruisme, de la pitié et 
de l'abnégation, valeurs dont Nietzsche montre qu'elles ont été autant de moyens permettant aux plus faibles de désarmer ceux qu'ils craignaient et de s'en protéger ( $c f$. par ex. Par-delà bien et mal $§ 201$ et FP XIV 15 [110]). Il indique également, par ailleurs, qu'une différence majeure semble séparer l'époque moderne des époques plus reculées. Celles-ci valorisaient explicitement la communauté au détriment de l'individu — au point d'ignorer même toute notion d'ego, de conscience ou de liberté individuelles au sens où nous l'entendons, et de considérer la mise à l'écart de la communauté comme la pire des condamnations. L'époque moderne au contraire affirme explicitement la valeur de l'individualité, l'importance du «sentiment de soi », du fait d'« être soi » et non pas seulement un élément du tout, et dans ce contexte encore la valeur de la solitude pour l'individu qui se doit de se retrouver et se connaître lui-même (Gai savoir § 117). Mais cette découverte moderne de l'ego constitue-t-elle vraiment le gage et la condition de possibilité d'une véritable solitude? Cela ne va, aux yeux de Nietzsche, nullement de soi, si l'individu qui prétend s'isoler pour mieux se retrouver emporte avec lui dans sa chambre solitaire ou son désert les valeurs « du troupeau ». Cela va à vrai dire d'autant moins de soi que la valorisation moderne de l'individualité se double comme on l'a vu d'une identification de la notion d'individu à celle de «sujet» pensant, et par là d'une tendance à penser les individus comme semblables par leur essence et leurs facultés (conscience, raison, volonté, etc.), et donc comme égaux en droit. C'est là précisément l'un des points caractéristiques des valeurs que Nietzsche désigne comme «démocratiques » et «grégaires»: on tient ici quoique «sans s'en rendre compte tous les ego pour équivalents » (FP X 25 [287]), on considère tout autre homme comme son prochain et son semblable. De façon paradoxale, la survalorisation de l'individu, de l'ego, conduit pour finir à considérer qu' «qu'à peu près tout le monde a la même valeur » (FP XIV 14 [5]). Nietzsche dénonce en conséquence l'individualisme moderne comme n'étant 
que le masque d'une volonté d'uniformiser l'humanité, comme une manière détournée de mieux affirmer la valeur de l'homme général, de «l'homme-moyenne», au détriment de toute authentique singularité (cf. Par-delà bien et mal § 201).

Plus précisément encore, Nietzsche indique que l'on prétend ici penser la possibilité d'une volonté libre, et par là d'une libre pensée qui requerrait seulement de faire le choix rationnel de l'indépendance à l'égard des opinions et des croyances admises. Mais si cette idée de volonté libre n'est qu'une croyance issue de besoins plus profonds que l'on se garde de jamais interroger, alors l'indépendance que l'on prétend fonder sur elle ne sera jamais que le masque des valeurs et des croyances communes. Celui qui croit parler de sa voix propre en revendiquant sa liberté individuelle ne se fait jamais que l'écho de la foule. L'individu dit ici, quoique sans le savoir, sa dépendance à l'égard des valeurs du troupeau dans l'instant même où il proclame hautement son autonomie, comme le montre l'analyse que conduit Nietzsche à l'égard des « libre penseurs » : ceux-ci ne prétendent s'affranchir de tout dogmatisme que pour mieux céder au préjugé commun concernant la valeur de la raison, et de l'égale valeur de tout individu doué de cette faculté. Les « libres penseurs » ne sont pas encore des « esprits libres », parce qu'ils ne sont encore que les «porte-parole des "idées modernes" » et restent de ce fait «sans solitude, sans solitude à eux » (Par-delà bien et mal § 44). De cette contradiction inhérente à la modernité qui ne proclame l'importance de l'individualité et de l'indépendance que pour mieux les réduire —, témoignent nettement selon Nietzsche les soupçons, voire les calomnies qui continuent immanquablement de s'élever contre celui qui en viendrait à contester effectivement ces valeurs admises, et qui dès lors se trouverait véritablement seul face à tous : «l'isolement » reste pour finir « l'argument qui anéantit jusqu'aux meilleurs arguments en faveur d'une personne ou d'une cause! - Ainsi parle, du fond de nous-même, l'instinct du troupeau » (Gai savoir § 50). 
Ainsi, c'est à l'exigence d'une radicalité nouvelle dans la réflexion sur la solitude, et quant à la possibilité d'une solitude effective — parfois désignée comme une « solitude propre », une « solitude à soi »— que nous confronte Nietzsche. Ni l'isolement spatial, ni la proclamation d'une liberté ou d'une autonomie individuelles, ne sont en effet suffisants à cet égard, puisqu'ils ne témoignent nullement d'un affranchissement, mais au contraire d'une profonde dépendance à l'égard des valeurs actuelles. Il est en effet possible, paradoxalement, de penser et de vivre la solitude d'une manière qui aux yeux de Nietzsche demeure fondamentalement grégaire. Avoir une « solitude à soi », comme l'indique de manière négative l'exemple des « libres penseurs », implique un questionnement radical quant aux valeurs, c'est-à-dire aux préférences inconscientes, qui sous-tendent usuellement la valorisation de l'indépendance et de la solitude mêmes, et ainsi quant à la conception de ces dernières. Ce questionnement — généalogique sur les valeurs qui conditionnent nos manières habituelles de penser implique de ne pas prétendre d'emblée les nier ou s'en abstraire; mais au contraire de considérer avec attention, en philosophe-généalogiste et en philosophe-médecin, ces valeurs et l'ensemble des symptômes auxquels elles donnent lieu. Or, un tel questionnement suppose manifestement que le philosophe continue d'être en quelque façon en relation avec cela même qu'il interroge - autrement dit avec ses semblables et sa culture, s'il veut éviter de se contenter d'une solitude illusoire.

\section{« La solitude de l'un est la fuite du malade... » : l'évaluation des solitudes}

Bien loin de devoir renoncer à toute relation, le philosophe et esprit libre doit tout au contraire avoir la force de prendre acte du réseau de relations dans lequel il se trouve d'abord nécessairement pris. C'est ce qui explique que Nietzsche fasse régulièrement usage de formulations énoncées à la première personne du pluriel («nous...»), qui indiquent qu'il entend 
s'inscrire, ou du moins prendre acte de ce qu'il s'inscrit d'abord nécessairement, dans une ou des communautés déterminées : celle des «hommes » d'abord (Humain, trop humain I § 16), mais plus précisément aussi celle des hommes «actuels » (Aurore $\S 9$ et $\S 154$; Gai savoir, § 18, $\S 152$, etc.) ou «modernes » (Gai savoir § 356, § 372, § 379; Par-delà bien et mal § 215, § 224, etc.), héritiers d'une longue histoire culturelle; celle des « Européens » (Gai savoir, § 346, § 352; Par-delà bien et mal $\S 224$, etc. $)^{16}$, et par là même celle des hommes « décadents » soumis aux valeurs du platonisme et du christianisme (Le cas Wagner, avant-propos); celle des «savants » (Par-delà bien et mal VI), des hommes « assoiffés de raison » (Gai savoir § 319) et de vérité — mais qui pour cette raison même se retournent pour finir contre la survalorisation de la raison et de la vérité; celle aussi, donc, des hommes pris dans ce nécessaire mouvement de dévalorisation des valeurs que Nietzsche nomme le «nihilisme », et qui peut être source d'un désespoir absolu pour beaucoup, ou l'indice de la possibilité d'une nouvelle aurore et d'un nouvel avenir, et par conséquent d'un «gai savoir » pour d'autres (§ 343). Cet usage récurrent et multiple du « nous » a en vue d'indiquer que l'indépendance du philosophe ne lui est pas d'emblée donnée, mais ne peut que se conquérir à partir d'une appartenance première à une communauté (humaine, moderne, européenne...). Elle implique en d'autres termes que le philosophe reconnaisse d'abord, non seulement qu'il est un élément de la multitude, mais plus radicalement aussi qu'il porte en lui les valeurs dont les Européens sont nécessairement les héritiers.

Sa capacité à interroger ces valeurs ne tient donc pas à ce qu'il aurait d'emblée la force de s'en abstraire, mais tout au contraire à deux points essentiels. Elle vient, d'une part, de ce que lui-même vit effectivement selon ces mêmes valeurs, et vit alors aussi le mouvement (nihiliste) d'autodépassement auquel doit les conduire leur nature même. Mais elle est d'autre part rendue possible, plus précisément, par le fait qu'il porte en lui cette caractéristique également propre à la 
culture européenne moderne qu'est le «sens historique »(Par-delà bien et mal § 224), c'est-àdire une tendance spontanée à envisager aussi toute la diversité des cultures et des valeurs passées, qui lui permet de tisser des liens et d'ouvrir des perspectives multiples qui rendront possible un écart et une distance à l'égard des valeurs qui sont d'abord les siennes, et par là enfin l'aperception du caractère problématique de celles-ci ( $c f . \S 186)$. Si tous les hommes modernes ne sont pas des esprits libres, c'est que le type du philosophe dispose d'une capacité de maîtrise, de comparaison, d'évaluation et — on y reviendra — de hiérarchisation et de sélection de cette diversité $^{17}$, qui fait défaut à la plupart.

C'est dans ce contexte que doivent également être comprises certaines des métaphores grâce auxquelles Nietzsche thématise l'indépendance du philosophe : les cimes, les perspectives élevées, ne sont pas synonymes d'une absence de toute relation, mais bien d'une distance qui implique encore un mode de relation spécifique. C'est le caractère pluriel des relations, c'est-àdire un rapport multiple à l'altérité, qui rend possible ici la mise à distance, et ainsi la mise en question et l'évaluation de ce qui nous était le plus propre et le plus proche : «On n'est philosophe qu'à l'étranger », écrit Nietzsche en ce sens, « et le philosophe doit ressentir comme étranger ce qui lui était le plus proche » (FP Considérations inactuelles I-II 23 [23]). C'est un propos semblable que développe encore le $\S 441$ d'Aurore: «Pourquoi les choses les plus proches nous deviennent toujours plus lointaines. - Plus nous réfléchissons à tout ce qui a été et sera, plus ce qui est, précisément aujourd'hui, devient pâle. Si nous vivons avec des morts et si nous mourons de leur mort, que sont désormais pour nous les êtres "les plus proches"? Nous devenons plus solitaires - mais c'est parce que le flot entier de l'humanité bruit autour de nous. » Être loin de l'autre n'est pas être tout à fait sans l'autre : la solitude n'est pas disparition de toute relation, mais elle suppose d'abord au contraire une multiplication des relations (« le flot 
entier de l'humanité bruit autour de nous »). Une telle multiplicité rend possible l'appréhension à la fois du propre dans sa différence et de l'altérité dans sa spécificité. La distance qu'elle engendre n'est nullement ce qui autorise à ne plus voir, à ignorer, mais au contraire ce qui permet de voir mieux et davantage — tout comme l'éloignement permet parfois de mieux juger de l'ami que, trop présent ou trop proche, nous ne savions plus apprécier ni considérer ( $c f$. Aurore $§ 485$ et Humain, trop humain I § 428). La diversité et ainsi la distance du regard et des perspectives qui caractérisent le philosophe sont alors aussi ce qui lui permet de voir la solitude comme problème - comme n'étant pas une, mais multiple, et susceptible d'être envisagée comme un symptôme dont la valeur et le sens (comme c'est le cas de tout symptôme, qui ne fait jamais sens isolément) ne sont nullement univoques.

Pour cette raison, on découvre dans les écrits de Nietzsche une manière d'évoquer la solitude qui peut apparaître dans un premier temps instable, voire contradictoire. Elle est en effet tantôt thématisée comme une qualité et une vertu des hommes les plus puissants, comme source de «bienfaits », ou comme un possible remède à certains états maladifs ( $c f$. Aurore $§ 114$ et $\S 202)$; et tantôt décrite et critiquée comme une cause ou un symptôme de maladie, comme l'un des traits caractéristiques des hommes les plus faibles, qui vivent selon des valeurs ascétiques. Comme le dit en d'autres termes Zarathoustra («Sur le mont des oliviers ») : si la solitude peut être parfois «fuite devant le malade», il peut cependant arriver aussi qu'elle soit « la fuite du malade », c'est-à-dire la fuite de celui qui n'a plus la force d'affronter ce qui est autre, d'interroger la diversité qui est non seulement hors de lui, mais en lui. La solitude, qui est ici expression d'un renoncement, est synonyme d'un enfermement maladif loin du monde que l'on n'a plus la force d'affronter, et par conséquent un symptôme de faiblesse (Aurore $\S 440$; FP Aurore 4 [46]). Elle peut aller de pair également avec un besoin de se faire souffrir, de se 
torturer soi-même là où le degré de force est si faible qu'il ne peut plus s'extérioriser, mais seulement se retourner contre lui-même. Une telle solitude est alors l'une des caractéristiques de ce que Nietzsche critique dans Par-delà bien et mal sous le nom de «névrose religieuse », qui se trouve toujours « liée à la prescription de trois régimes dangereux : solitude, jeûne et abstinence sexuelle » (Par-delà bien et mal § 47; cf. déjà Humain, trop humain I, § 47). Mais Nietzsche soutient également le même propos à l'égard de certains penseurs qui ont prétendu s'isoler du monde, et parfois aussi à l'égard de lui-même : la solitude qui se conçoit avant tout négativement comme retrait hors du monde, comme mode de défense à l'égard d'une altérité que l'on n'a pas la force de considérer, est une solitude «stérile », source de «mélancolie » (Aurore $§ 440$ ), elle est l'une de ces « humeurs mauvaises » (Humain, trop humain I, préface $\S 2$ ) qui constituent un danger pour le penseur. C'est pourquoi il faut distinguer deux grands types au moins de solitude philosophique (dont chacun est susceptible de recouvrir des degrés variés), qui ne se ressemblent que superficiellement.

\section{La solitude et le danger de l'isolement}

Le premier type de solitude philosophique a pour source un besoin, issu de la faiblesse et de la crainte, de fuir le monde, de se soustraire à toute relation, à toute attaque et à toute lutte. Ce fut selon Nietzsche le cas par exemple de Spinoza, ce «malade érémitique » qui avait besoin de présenter sa philosophie sous une forme capable d' « inhiber le courage de l'assaillant » afin de la défendre de toute attaque (Par-delà bien et mal §6), et pour lequel l'exil et l'isolement contraints constituent encore un moyen de se présenter comme un vénérable «martyre » de la vérité, comme le «défenseur de la vérité sur terre » $(\$ 25)$. Mais on voit alors qu'en cet habile retrait Spinoza n'en continue pas moins de demeurer soumis à des valeurs communément 
admises (la croyance à la vérité, au caractère absolument vénérable de celle-ci). La relation de Nietzsche à Spinoza ne s'épuise pas dans cette critique : nous y reviendrons plus loin. Mais quelles que puissent être par ailleurs l'originalité et la force de sa pensée, il n'en cède pas moins à une forme de solitude qui demeure selon Nietzsche insuffisante (puisque le solitaire demeure soumis ici à certains préjugés communs) et affaiblissante (puisqu'en se soustrayant à toute relation, le penseur s'épargne l'effort de surmonter les attaques et les difficultés nouvelles qui pourraient surgir). Mais il faut surtout resituer cet exemple dans un contexte plus général. Nietzsche remarque en effet par ailleurs qu'un tel isolement maladif est généralement la conséquence nécessaire de la survalorisation de la sphère intellectuelle, considérée comme autonome, et dont on préfère ignorer les sources vitales et le caractère hérité : en se concevant comme âme ou comme esprit, et en considérant — comme le fait précisément entre autres Spinoza ( $c f$. Par-delà bien et mal $\S 37$ et $\$ 333$ ) — la pensée comme pure et désintéressée, l'individu est conduit à ignorer tout ce qui nécessairement le lie à sa communauté et aux autres (à ignorer, en d'autres termes, le caractère toujours déjà incorporé des valeurs communes), et il croit à tort que s'isoler de tous permet d'être véritablement seul. Le manque de subtilité de cette conception conduit à une conception excessive de la solitude comme isolement. «Prudence à l'égard de 1'“intellectualité" », écrit Nietzsche en ce sens dans un fragment posthume : «elle corrompt le caractère, en rendant extrêmement solitaire [extrem einsam] : solitaire, c'est-à-dire non lié, détaché » (FP XIII 11 [80]).

Le second type de solitude que Nietzsche met en évidence s'inscrit dans un tout autre contexte et émane de tout autres besoins. Il acquiert par là aussi un tout autre sens : Nietzsche évoque en ce sens la «bonne solitude, la solitude libre, malicieuse, légère » (Par-delà bien et mal § 25) qui n’est donc pas simple renoncement au monde ou défense réactive à l'égard de ce 
qui est autre que soi. Une telle solitude (Einsamkeit) se distingue dès lors de l'isolement (Vereinsamung) maladif qui consiste à se retirer en soi-même et à prétendre ignorer ce qui est autre. Si elle est «bonne », c'est justement parce qu'elle est « libre » et « légère », c'est-à-dire parce qu'elle ne se conçoit pas comme enfermement en soi-même, comme indépendance illusoire à l'égard de l'altérité qui est toujours déjà en soi, mais que l'on se refuse à considérer. Et c'est pourquoi, s'il est vrai comme on l'a vu que «[t]out homme hors du commun aspire instinctivement à sa citadelle et sa retraite secrète où il soit délivré de la foule », ce propos se doit cependant d'être complété et précisé. Car le penseur authentique se doit aussi d'avoir la force de ne pas se «dérobe[r] à tout jamais » à la foule, et de ne pas seulement «demeure[r] [...] calmement et orgueilleusement caché au fond de sa citadelle ». Il se doit de fréquenter ceux et ce qu'il prétend étudier, et pour cela il lui faut aller parfois «vers le bas, et surtout "au beau milieu” » (§ 26). Telle est précisément l'exigence qui manifestement anime d'abord — et rythme régulièrement ensuite - le voyage de Zarathoustra qui quitte d'abord sa patrie pour jouir de la solitude, mais qui, lassé de celle-ci et de sa «sagesse », éprouve finalement le besoin de « décliner » et de « descendre » vers les hommes (Ainsi parlait Zarathoustra, prologue §1).

Cela revient à dire que la solitude — si elle reste conçue avant tout comme retour à soi et retrait en soi-même — n'a pas de valeur en soi ni n'est une fin en soi : «Dans la solitude grandit ce que chacun y apporte, même la bête intérieure. De sorte que la solitude est à déconseiller à beaucoup » («De l'homme supérieur » § 13). Lorsque la solitude n'est qu'un moyen de défense, lorsqu'elle se trouve convertie en un simple isolement, elle devient «poison » et aggravation du mal pour celui qui est déjà affaibli et malade (Gai savoir $§ 359)^{18}$. Et c'est pourquoi, à l'inverse, la solitude que pense Nietzsche ne suppose une distance et un écart que pour mieux penser aussi la possibilité d'une (nouvelle) rencontre. Si une telle conception peut sembler paradoxale, il faut 
voir que sa nécessité et sa cohérence viennent de ce qui a été d'abord montré à propos de l'individu : si celui-ci porte toujours déjà en lui les valeurs communes, alors la prétention à un détachement immédiat ne peut être qu'illusoire. C'est à l'inverse, comme on l'a montré, la considération de type d'hommes et de valeurs variés qui permettra seule de se mettre à distance de ceux auxquels on était d'abord lié, et le cas échéant de lutter aussi contre eux pour s'en affranchir.

C'est pourquoi Nietzsche insiste encore sur un point important. Si la solitude est nécessaire, elle implique cependant toujours un grave risque. Celui-ci consiste en ce que, croyant se déprendre de ce qui est autre, on se rende par là dépendant de soi, c'est-à-dire d'une perspective unique qui, là même où elle serait authentiquement singulière, n'en pourrait pas moins demeurer indûment limitée et insuffisante. Pour cette raison, Nietzsche indique encore que le penseur à l'esprit libre ne doit pas demeurer « en soi ». Comme on l'a vu plus haut, c'est bien parce qu'il a eu le courage de ne pas rester toujours «chez soi » que le penseur peut désormais considérer d'un œil nouveau les choses qui lui étaient « le plus proches » (Humain, trop humain I, préface $§ 5$ ). Être, aller hors de soi est la condition de la découverte et surtout de la maîtrise de soi. Car ce n'est qu'à cette condition qu'il est possible d'examiner, d'évaluer, le cas échéant de tenter de modifier ou de surmonter ce qui est en soi $(\S 6)$.

La solitude ne se confond donc pas avec une simple disposition à demeurer en soi et à «jouir de soi », mais elle est au contraire un appel à l'affranchissement, au dépassement de soi, et elle suppose pour cette raison l'exigence d'une confrontation avec ce qui est autre (FP IX 16 [86]) : «Appel à la solitude et à l'affranchissement de soi! » (FP IX 4 [230] $)^{19}$. C'est en ce sens que Nietzsche indique dans le Voyageur et son ombre (§ 306) que «[1]orsqu'on est arrivé à se trouver soi-même, il faut s'entendre à se perdre de temps en temps — pour se retrouver ensuite : 
en admettant, bien entendu que l'on soit un penseur. Car il est préjudiciable à celui-ci d'être toujours lié à une seule et même personne. » Une authentique solitude, gage d'une véritable indépendance, implique de ne pas demeurer lié à qui que ce soit — fût-ce à soi-même, ce pourquoi elle implique la confrontation à l'autre, la recherche même d'adversaires contre lesquels il faille continuer de lutter afin d'exercer et de développer ses propres forces (Aurore $\S 542$ ). La relation aux autres n'est pas toujours synonyme de perte de soi, et c'est parfois l'attachement à soi-même qui rend impossible une liberté authentique, comme l'indique encore Zarathoustra : «L'un va auprès de son prochain, parce qu'il se cherche lui-même, et un autre parce qu'il aimerait se perdre. Votre mauvais amour pour vous-même fait pour vous de la solitude une prison » («De l'amour du prochain »). Nietzsche peut alors énoncer en ces termes, dans le $\$ 41$ de Par-delà bien et mal, la spécificité de son exigence de solitude et d'indépendance :

On doit se mettre à l'épreuve pour se prouver que l'on est destiné à l'indépendance et au commandement; et ce en temps voulu. On ne doit pas esquiver ces épreuves, bien qu'elles soient peut-être le jeu le plus dangereux que l'on puisse jouer [...]. Ne pas rester lié à une personne : et ce quand bien même elle serait la plus aimée, — toute personne est une prison, un réduit également. Ne pas rester lié à une patrie : et ce quand bien même elle serait en proie à la plus grande souffrance [...]. Ne pas rester lié à une science : quand bien même elle séduirait par les découvertes les plus précieuses [...]. Ne pas rester lié à sa propre rupture [nous soulignons], à cette voluptueuse distance et étrangeté de l'oiseau qui s'enfuit toujours plus haut pour voir toujours plus au-dessous de lui : - le danger de la créature ailée. Ne pas rester lié à nos propres vertus et devenir, comme totalité, victime de quelqu'une de nos particularités [...]. On doit savoir se préserver : la plus forte mise à l'épreuve de l'indépendance. (Par-delà bien et mal § 41)

On voit ici que la solitude que pense Nietzsche n'est pas un état, ou une constante manière d'être. Elle se conçoit plutôt comme disposition ou comme aptitude, une «aptitude à rester isolé et à répondre de soi » (\$210), en soi aussi bien que face à d'autres. Elle est mouvement de rencontre puis de mise à distance et elle implique un constant processus d'appropriation et de 
distanciation. L'homme doué d'un haut degré de force est celui qui est capable de ne rien fuir d'emblée, mais qui a néanmoins la force pour finir de «refus[er] de se laisser approcher » (§ 230) par ce qu'il considérera comme de peu de valeur. Il est un «principe de sélection » (Ecce homo, «Pourquoi je suis si sage » §2), c'est-à-dire qu'il est capable à la fois d'affronter une grande diversité (d'hommes, de cultures, de valeurs) pour l'évaluer et de la hiérarchiser afin de sélectionner ce qui a le plus haut degré de valeur et de mettre au contraire à l'écart ce qui pourrait être source d'affaiblissement — de manière à «se préserver » de ce qui porterait atteinte à sa force et à son indépendance mêmes ${ }^{20} . \mathrm{Si}$, dans cette perspective, Nietzsche indique parfois qu'il faut en effet apprendre à l'individu à savoir s'isoler afin qu'il apprenne par là à se préserver de certaines sollicitations, une telle isolation ne peut cependant être pensée que comme «temporaire », que comme condition et moyen d'une appréhension et d'une sélection plus fine de ce qui est autre : «La formation de la personne requiert l'isolation [Isolirung] temporaire, la contrainte à une existence défensive et armée, quelque chose comme l'emmurement, une plus grande force de retranchement; et avant tout une impressionnabilité bien moindre que n'en a l'homme moyen » (FP XIII 10 [59], trad. mod.).

La solitude doit être pensée en ce sens comme une constante « mise à l'épreuve », comme une «expérimentation », comme un Versuch que le philosophe conduit à l'égard de lui-même et qui se constitue de deux moments à vrai dire indissociables l'un de l'autre : il s'agit à la fois d'avoir la force de prendre ses distances d'avec ce à quoi l'on est d'abord attaché et de se confronter aussi à l'altérité et à la nouveauté pour mieux revenir à et mieux s'affranchir de soi et ainsi de ne pas même demeurer «lié à sa propre rupture». La solitude ne peut donc être assimilée à un «exil » que dans la mesure où elle est aussi un «voyage » qui n'implique de quitter une culture et une « patrie » que pour en rencontrer et s'adapter à d'autres, et pour mieux 
parvenir à la penser et à l'évaluer en retour - ce dont la figure nietzschéenne du «bon Européen » se trouve être précisément l'incarnation (cf. Par-delà bien et mal § 242). Tout comme le penseur est «inactuel» — non dans la mesure où il ignore absolument ce qui est «actuel », mais parce que sa connaissance de la variété des époques passées lui permet de considérer autrement l'« actualité » que ne le font ses contemporains —, de même le philosophe est «solitaire », non parce qu'il s'isolerait radicalement de l'humanité, mais parce que considérant l'humanité dans toute sa variété, il en vient à la penser et à l'évaluer autrement que ne le font la plupart des autres hommes, et parce qu'il parvient aussi par là à s'affranchir de ce à quoi il était initialement asservi. En dernier lieu, le philosophe devenu authentiquement solitaire — c'est-à-dire celui qui aura acquis la force de se déprendre effectivement de l'autorité des valeurs communes — le sera aussi bien dans le désert qu'au milieu d'une foule : où que vive cet homme véritablement solitaire et « insolite », qui « rentre en contradiction avec les formes et les ordres existants », « le désert et la caverne sont déjà là »(Considérations inactuelles III, § 3).

Mais il faut aller plus loin encore : parce que la tâche du philosophe n'est pas seulement d'ordre théorique, mais qu'elle implique à terme également et surtout un enjeu pratique — parce que cette considération nouvelle de l'humanité fait surgir pour finir la nécessité de transformer et d'élever (züchten) l'humanité et de rendre possible surtout l'avènement d'un type humain supérieur, le philosophe ne saurait davantage prétendre s'affranchir entièrement de ses semblables que le médecin ne pourrait prétendre exercer son art en l'absence de tout patient. Or, le philosophe se trouve ici confronté à un problème : car quoique voulant être solitaire, et non pas isolé, il se trouve d'abord condamné par force, du fait de la nouveauté et de l'originalité de sa position, à un nécessaire isolement qui semble rendre vaines sa tâche et ses attentes, et stérile aussi sa solitude même. 


\section{«Nous, ermites » : du danger de l'isolement à la nécessité d'une communauté}

Le philosophe que pense Nietzsche - mais aussi manifestement le philosophe Nietzsche lui-même — semble en effet être pris dans une situation de double bind, de «double contrainte ». Il désire, d'un côté, que son propos soit entendu, afin qu'il puisse avoir quelque effet sur l'humanité, ou du moins sur le public européen moderne qu'il cherche à transformer et à guérir de la maladie nihiliste issue des valeurs ascétiques. D'un autre côté, toutefois, le caractère original de son propos, qu'il ne peut vouloir simplifier ou rendre commun sans le trahir par là même, le conduit nécessairement à être mis à l'écart de cette communauté qu'il veut transformer et le rend même incompréhensible pour la plupart des membres de cette dernière. Comment le philosophe-médecin peut-il donc jouer son rôle à l'égard d'un «patient » qui se détourne de lui ou simplement l'ignore? Comment cette forme particulière d' « amour » envers l'homme, qui caractérise le philosophe (Par-delà bien et mal § 295) — ce «grand amour» qui dans la perspective de Nietzsche ne doit exclure ni la distance, ni la dureté, ni la cruauté (Généalogie de la morale III, § 24) —, peut-elle effectivement s'exercer? Comment, en d'autres termes encore, peut s'exercer ce que Zarathoustra désigne comme la «vertu qui prodigue » et qui suppose en effet que « la hauteur solitaire » du penseur «ne soit pas éternellement vouée à la solitude et ne se contente pas éternellement d'elle-même » (« Des trois maux » § 2)?

C'est dans ce contexte qu'il faut comprendre le souci de la réception et de la possible mais nécessairement difficile — compréhension de ses écrits, qui est constamment présent chez Nietzsche, et ce dès la Naissance de la tragédie, dont la dédicace adressée à R. Wagner indique précisément déjà ce besoin de trouver, si ce n'est un public nombreux, du moins un ou quelques lecteurs susceptibles de l'entendre ${ }^{21}$. La troisième Inactuelle surtout mettra ensuite en évidence cette difficulté inhérente au projet philosophique qui est celui de Nietzsche, au travers de 
l'évocation de la figure de Schopenhauer, dont le caractère inactuel fonde «la véritable solitude », mais qui se trouve dans le même temps condamné à l'isolement (Vereinsamung) puisque, quoique lu par un grand nombre de lecteurs, il est demeuré incompris de tous, et par conséquent privé de tout ami véritable (Considérations inactuelles III $\S 3$ et $\S 7$ ). Or, un tel isolement constitue, nous dit Nietzsche dans ce même texte, un grave danger - le «premier danger », dont découlent tous les autres (désespoir, dépérissement et stérilité) — pour le philosophe : non seulement parce que les «solitaires » eux-mêmes ont «besoin de compagnons avec qui ils puissent se montrer ouverts et francs comme envers eux-mêmes » (§3); mais aussi et peut-être surtout parce que l'activité philosophique implique à terme une dimension qui n'est pas seulement théorique et critique, mais aussi pratique, éducative et culturelle, et en conséquence un «cycle de devoirs » dont Nietzsche nous dit (§ 5) qu'ils « ne sont pas les devoirs d'un homme isolé; on participe, bien au contraire, avec eux d'une puissante communauté dont les liens ne sont nullement des formes et des lois extérieures, mais une pensée fondamentale. C'est la pensée fondamentale de la culture », qui engendre le désir de former et de faire advenir ce que Nietzsche appelle à cette époque de «grands hommes » (§ 6) et qu'il désignera ultérieurement comme un «type supérieur» ou un type «relativement surhumain» (Ecce homo, «Pourquoi je suis un destin » §5). Or une telle tâche ne saurait être accomplie par un «homme isolé ». Elle rend nécessaire de se donner des «compagnons », elle implique, comme le montre la suite du même ouvrage, que la figure unique du solitaire laisse place à celle, plurielle, «des solitaires », et ce pour plusieurs raisons qu'il nous faut alors éclairer davantage.

La première consiste en ce que la relation amicale apparaît à Nietzsche comme constituant, sinon une condition, du moins un soutien pour la capacité d'indépendance ellemême. Celui qui a entrepris de penser contre cela même dont il dépend d'abord se trouve sans 
cesse soumis au risque, soit de céder à nouveau aux préjugés et aux valeurs qu'il a appris à questionner, soit de se détruire lui-même ( $c f$. Tongeren 218 sq.) dans la mesure où il se trouve d'abord privé de tout appui. Dans ce contexte, l'interlocuteur amical, l'existence de «pairs » qui le comprennent et lui répondent — le cas échéant, en s'opposant à lui de manière à lui permettre de poursuivre ou d'approfondir sa recherche —, peuvent être pensés comme un « rempart » qui lui permet de conserver ses distances à l'égard de la foule autant qu'à l'égard de lui-même et du désespoir qui parfois le guette ${ }^{22}: \ll$ je devrais avoir autour de moi un cercle d'êtres profonds et tendres », écrit en ce sens Nietzsche dans un fragment posthume, «qui me protègeraient un peu de moi-même et sauraient également m'égayer : car pour un être qui pense le genre de choses que je dois penser, le danger de se détruire soi-même est toujours imminent » (FP XII, 1 [1]). La relation amicale apparaît alors paradoxalement comme une condition utile au maintien d'une véritable solitude, ainsi que pourrait le confirmer une lettre adressée par Nietzsche à Rohde le 16 juin 1869 : «lorsque nous sommes ensemble, en vérité nous ne constituons pas une dyade, mais bien une véritable et authentique monade : nous sommes alors plus que jamais solitaires et coupés de tout le monde importun. »

Mais là ne s'arrêtent pas les raisons qui rendent nécessaires l'établissement d'une telle communauté. Elles tiennent en effet aussi à la nature de la tâche généalogique dans son double versant — axiologique et créateur —, qui doit être celle du philosophe. Cette tâche se caractérise par sa complexité, puisque le philosophe se doit d'enquêter sur la diversité — géographique aussi bien qu'historique - des cultures et des valeurs. L'ampleur d'une telle enquête, que Nietzsche compare dans le $\S 45$ de Par-delà bien et mal à une «grande chasse », doit nécessairement conduire le penseur à souhaiter « quelques centaines d'assistants de chasse et de fins limiers bien entraînés qu'il pourrait lâcher dans l'histoire de l'âme humaine pour y rabattre 
son gibier » et à regretter leur notable absence : «que de fois il lui faut se dire avec désespoir : “Un seul et unique homme! Ah, rien qu'un seul! et toute cette forêt, toute cette forêt vierge!'”...» Il lui faut, comme le dira encore le $\$ 7$ de la préface à la Généalogie de la morale ${ }^{23}$, « rechercher des compagnons savants, audacieux et à qui le travail ne fasse pas peur », afin de parvenir à «parcourir le formidable pays de la morale, lointain et si caché — de la morale qui a réellement existé, réellement été vécue — avec des questions absolument neuves ». Mais Nietzsche le remarque aussitôt, le caractère absolument neuf et donc difficile à comprendre de ce questionnement est précisément cause de ce que sa recherche de tels compagnons est jusqu'ici demeurée vaine : «je continue à le faire aujourd'hui ».

Or, ce n'est pas seulement l'enquête historique et psychologique qui requiert le soutien de tels compagnons. À terme, c'est aussi le projet d'un renversement des valeurs qui ont dominé jusqu'ici, qui suppose que leur soient substituées des valeurs nouvelles plus propres à élever l'homme, qui implique que le philosophe ne demeure pas isolé. Non seulement en effet cette tâche requiert une autorité qui peut difficilement être dévolue à un seul — et ce d'autant plus que comme on l'a vu, «l'isolement» est un «argument» qui permet à la foule de condamner le philosophe et de l'ignorer (Gai savoir § 50). Mais il faut dire en outre qu'une telle tâche requerra nécessairement une longue durée, ce qui suppose que le philosophe trouve des disciples qui soient susceptibles de la poursuivre sur un très long terme. C'est ce qui explique, d'une part, que l'esprit libre en appelle à des disciples et des continuateurs ${ }^{24}$, plus spécifiquement aussi à des «philosophes de l'avenir », à des «philosophes nouveaux » (Par-delà bien et mal $§ 42-\S 44$, $\S 203$ ) qui soient susceptibles d'appliquer effectivement à l'avenir les exigences qu'il aura mises en évidence. Cela explique également, d'autre part, que Nietzsche s'attache à faire de ses écrits le moyen d'une découverte de «pairs » susceptibles d'accompagner et de poursuivre sa tâche — 
tentative qui demeure cependant largement lettre morte, comme il le rappellera dans Ecce homo («Pourquoi j’écris de si bons livres »; cf. Par-delà bien et mal § 1) :

Une fois accomplie la partie de cette tâche qui consistait à «dire oui », restait celle de « dire non », de «faire non »: l'inversion des valeurs qui avaient cours jusqu'alors, la grande guerre [...]. Cela comportait aussi la lente quête d'êtres qui me fussent proches, d'êtres assez sûrs de leur force pour me prêter la main dans mon œuvre de destruction. Depuis lors, tous mes écrits sont des hameçons [...] Si rien ne s'est laissé prendre, la faute n'est pas mienne. Ce sont les poissons qui manquaient...

Nietzsche affirme bien ici tout à la fois la nécessité, et la difficulté, voire jusqu'ici l'impossibilité pour le philosophe de s'inscrire dans une communauté d' «êtres proches » sans lesquels sa tâche ne saurait que malaisément se poursuivre. Le propos est ici toujours double: une telle communauté est désirée, exigée, et cependant toujours et encore absente, tout au plus encore à venir, parce que les êtres authentiquement indépendants, et véritablement solitaires, sont l'exception et non la règle. C'est pourquoi les conséquences de ce propos se déploient également, dans les écrits de Nietzsche, suivant une double direction.

Faute d'une communauté présente et effective, Nietzsche entend d'une part penser l'existence d'une communauté que l'on pourrait dire «inactuelle», au double sens du terme. Cette communauté doit en effet être pensée relativement au passé et à l'histoire, au sein desquels le penseur peut découvrir de « grands individus » qui ont en quelque manière préfiguré sa propre recherche et sa propre volonté créatrice, et dont le rappel doit l'encourager dans sa tâche, comme le pourraient faire des compagnons vivants. Nietzsche évoque déjà en ce sens, dans la troisième Considération inactuelle, cette «République de génies » qui vivent «dans une simultanéité temporelle ». En s'inscrivant à son tour dans cette lignée, le penseur peut plus aisément se déprendre du présent et du chœur bruyant de la foule, pour perpétuer à son tour «le haut dialogue des esprits ». Le nécessaire rapport du philosophe à l'histoire, son « sens historique », 
lui permettent précisément d'entretenir un tel rapport à de grandes figures du passé qui soutiennent sa tâche présente et à venir : «La tâche de l'histoire est de servir d'intermédiaire entre [ces génies], pour, ce faisant, constamment susciter et soutenir l'éveil de la grandeur» (Considérations inactuelles III §9). Et Nietzsche lui-même ne laisse pas de s'inscrire explicitement au sein d'une lignée d'ancêtres et de prédécesseurs, dont il fait également parfois des interlocuteurs privilégiés, comme c'est le cas par exemple dans l'aphorisme intitulé «La descente à l'Hadès » qui clôt les Opinions et sentences mêlées : certains grands penseurs du passé sont à ses yeux plus vivants que les vivants eux-mêmes, et c'est par eux — plutôt que par aucun de ses contemporains — qu'il entend, après avoir « longtemps marché seul », s’expliquer et se «faire donner tort ou raison». Faute de disposer d'une communauté actuelle, il faut s'inscrire dans une communauté posthume ${ }^{25}$ : un penseur «n'a pas besoin de société, sinon de temps à autre pour embrasser ensuite d'autant plus tendrement sa solitude; les morts lui tiennent lieu de vivants et même d'amis : et ce sont les meilleurs qui aient jamais vécu » (Aurore § 566). Une telle communauté n'est d'ailleurs pas seulement conçue par défaut, puisqu'elle est avant tout engendrée par le caractère historique de l'enquête philosophique. Un fragment préparatoire à Ainsi parlait Zarathoustra peut alors caractériser la figure du philosophe réformateur de la manière suivante : «Toujours plus solitaire et plus exclu [einsamer und verbannter] : toujours plus brûlant d'amour et d'élan vers les hommes. / Toutes les souffrances caractéristiques du réformateur, et ses consolations. / 1) Consolation : je m'attaque au prochain millénaire / 2) Je vis comme en d'autres temps : ma hauteur m'ouvre le commerce avec les solitaires et les méconnus de tous les $\mathrm{t}<\mathrm{emps}>»($ FP IX 9 [31]). La distance prise à l'égard des hommes de ce temps est solidaire du « commerce » entretenu avec «les solitaires et les méconnus de tous les temps », qui limite le danger de l'isolement auquel se trouve confronté l'esprit libre. Épicure, Montaigne, 
Goethe, mais aussi Platon et Spinoza, font partie de ces grands interlocuteurs que Nietzsche évoque régulièrement à cet égard, et dont il se proclame parfois aussi l'héritier. Dialoguer avec un autre ne signifie pas s'accorder en tout point avec lui; être un héritier ne revient pas à être un simple imitateur ou un épigone. Les critiques que Nietzsche formule, par exemple, à l'égard de tel aspect de la philosophie spinoziste n'excluent pas, mais au contraire supposent, qu'il le reconnaisse en effet comme un interlocuteur digne de ce nom : «Attaquer est de ma part une marque de bienveillance, le cas échéant de gratitude », nous rappelle Nietzsche dans Ecce homo («Pourquoi je suis si sage », §7). Nulle contradiction, donc, à ce que Nietzsche adresse des reproches parfois virulents à Spinoza, entre autres sur la question de la solitude et de l'isolement, tout en le présentant comme l'un de ces compagnons qui peuvent empêcher que sa solitude ne se mue en isolement, comme en témoigne une fameuse lettre à Overbeck datée de juillet $1881:$ : Je suis très étonné, ravi! J'ai un précurseur et quel précurseur! Je ne connaissais presque pas Spinoza. Que je me sois senti attiré en ce moment par lui relève d'un acte "instinctif". In summa : ma solitude [Einsamkeit] qui, comme du haut des montagnes, souvent, souvent, me laisse sans souffle et fait jaillir mon sang, est au moins une solitude à deux [Zweisamkeit]. »

Mais ce propos permet aussi de mieux comprendre pourquoi Nietzsche peut également sans incohérence proclamer son indépendance et sa solitude, et faire néanmoins parfois écho à certains de ses prédécesseurs sur la question même de la solitude : car l'indépendance de l'esprit libre n'est pas prétention à l'autogenèse, elle n'exclut pas de s'inscrire dans la continuité de penseurs dont on se proclame, non pas l'épigone, mais l'héritier, c'est-à-dire celui qui est capable non seulement de reprendre mais aussi de corriger et de poursuivre la pensée d'un prédécesseur. 
Toutefois, Nietzsche reconnaît qu'une telle communauté disparue ou posthume ne saurait suffire au penseur qu'oriente également le souci de l'avenir: il faut au philosophe des « compagnons vivants », et non pas seulement des «compagnons morts et des cadavres [qu'il] porte avec lui où [il] veut»(Ainsi parlait Zarathoustra, prologue § 9). Mais puisqu'une telle communauté présente et vivante n'est pas encore d'actualité, elle doit alors être forgée, imaginée, comme c'est le cas pour les «philosophes à venir » qu'évoque Par-delà bien et mal, mais déjà aussi pour les « esprits libres » que décrit la préface à Humain, trop humain (§ 2) :

Et c'est ainsi que j'ai inventé, un jour que j'en avais besoin, les « esprits libres » [...]: de ces « esprits libres », il n'y en a, il n'y en eut jamais, — mais, comme je l'ai dit, c'est leur société qu'il me fallait alors pour garder ma bonne humeur au beau milieu d'humeurs mauvaises (maladie, isolement, exil [...]) : braves compères de fantômes avec qui rire et bavarder quand on a envie de rire et bavarder, et que l'on envoie au diable s'ils deviennent ennuyeux, - en dédommagement d'amis qui vous manquent.

Cette imagination n'est pas ici seulement expression d'un regret et d'une impossibilité, mais également d'une exigence, d'une attente et d'un espoir :

Ces esprits libres, qu'il puisse y en avoir quelque jour, que notre Europe ait à l'avenir, parmi ses fils de demain et d'après-demain, de ces gais et hardis lurons, bien palpables en chair et en os, et non pas seulement, comme dans mon cas, en forme de spectres et de fantasmes au gré d'un anachorète, c'est bien moi qui serais le dernier à en douter. Déjà je les vois venir, lentement, lentement; et peut-être aurai-je fait quelque chose pour hâter leur venue quand j'aurai décrit par anticipation sous quelle étoile je les vois naître et par quels chemins arriver?...

Une telle communauté doit être imaginée et décrite si l'on veut pouvoir tenter de la réaliser : telle est précisément l'une des fonctions des écrits nietzschéens, lesquels ne visent pas seulement à dire une philosophie théorique, mais doivent aussi être considérés comme le moyen de sélectionner, le cas échéant de transformer, des lecteurs susceptibles de les appréhender et par là, de constituer cette communauté attendue. On comprend alors pourquoi le « je » de l'esprit libre et du philosophe solitaire en vient souvent à s'exprimer sous une forme plurielle : «nous, 
ermites »(Par-delà bien et mal $\S 230$ et $\S 283)$; «nous, immoralistes » (§ 26); «nous, bons Européens » (§ 241 et $\S 243)$; «nous, esprits libres »(Humain, trop humain I, préface $\S 7$; Gai savoir § 343; Par-delà bien et mal §44, § 203, § 227, § 230)... Le « nous » dont il est question ici, et qui se fait surtout prégnant à partir de 1886, diffère de celui qui a été évoqué dans la deuxième partie de cette étude : il a cette fois une fonction que l'on pourrait dire performative, plutôt que descriptive. Il vise à indiquer ce qui fait jusqu'ici défaut, ce qui doit encore être accompli. Il est appel à la constitution d'un tel «nous » et d'une telle communauté, de la part d'un « je » qui aperçoit clairement le risque de demeurer isolé et stérile, plutôt que véritablement solitaire.

\section{Conclusion}

La solitude que pense Nietzsche se conçoit paradoxalement comme impliquant toujours la multiplicité. Le solitaire n'est pas un, mais pluriel comme l'est tout individu, et il ne peut nier cette pluralité (et le rapport à l'altérité qu'elle implique) sans se nier lui-même et se perdre d'emblée dans la multitude à laquelle il prétendrait trop précipitamment échapper.

C'est pourquoi aussi la solitude se conçoit comme processus et comme épreuve, comme tension et point d'équilibre fragile entre deux dangers jamais écartés : celui de l'asservissement à ce qui est autre — et pour une part néanmoins déjà en soi; celui de l'attachement à un « soi » qui ne serait plus capable de prendre le risque d'affronter ce qui est autre pour se surpasser. Au sein d'une pensée qui se refuse à concevoir l'individu comme existant par soi, et à assimiler l'indépendance à une prétention (illusoire) à l'autosuffisance, la solitude ne peut se concevoir à l'exclusion de toute relation. Elle est au contraire, on l'a vu, constant processus de mise en relation, d'appropriation ou de mise à distance de ce qui est autre. Il faut le noter, pour finir : le 
«surhumain » lui-même ne se concevra pas indépendamment de la multitude, ni ne requerra sa disparition. Étant un type «supérieur», un «type relativement surhumain» (Ecce homo, «Pourquoi je suis un destin » $\$$; nous soulignons), c'est bien par relation avec ce qui est autre que lui qu'il doit d'abord être pensé, et c'est cette relation qui constituera encore la condition de possibilité de son avènement ( $c f$. Crépuscule des idoles, «Incursions d'un Inactuel » § 37; L'antéchrist §57). Tout comme l'existence de la règle est nécessaire au surgissement de l'exception, le maintien à l'existence de types humains plus faibles, et du type « grégaire » luimême, est nécessaire pour qu'apparaisse un tel « type supérieur ». S'il convient assurément de ne pas confondre le «type solitaire » et le «type grégaire », il faut néanmoins rappeler que «tous deux sont nécessaires » et surtout que «leur antagonisme est nécessaire » (FP XIII 10 [59]). Le type supérieur ne se caractérisera en conséquence ni par un isolement absolu, ni par une volonté d'imposer à tous ses exigences propres à la façon d'une loi, pour reconduire à soi tout ce qui est autre ( $c f$. Ansell-Pearson, « Nietzsche on Autonomy and Morality » 27). Parce que la pensée de Nietzsche se caractérise comme une pensée de la multiplicité, des différences et de la hiérarchie, la solitude du type surhumain, tout comme celle du philosophe, ne se concevra donc pas comme une négation de toute altérité, mais bien comme un constant travail de différenciation et de distanciation, qui suppose en effet que demeure toujours une relation à ce qui est autre. 


\section{Notes}

${ }^{1}$ Les textes de Nietzsche sont cités selon les Euvres philosophiques complètes (en 18 vol. chez Gallimard), à l'exception de ceux issus des ouvrages suivants : Crépuscule des idoles, Le gai savoir, Généalogie de la morale et Par-delà bien et mal.

${ }^{2}$ Contre le type de lecture qui postule l'existence de différentes «périodes », et par conséquent d'une évolution ou de ruptures au sein de la pensée de Nietzsche, nous soutenons qu'il existe au contraire une continuité fondamentale des écrits nietzschéens - continuité sur laquelle Nietzsche ne laisse pas lui-même d'insister, en particulier dans la préface à la Généalogie de la morale ( $\$ 2$ et $\S 8$ ). Celle-ci indique en effet que les modifications, lorsqu'elles existent, relèvent surtout du mode d'expression de cette pensée, soit du développement progressif de ce que Nietzsche lui-même considère comme son «nouveau langage », qui doit permettre de dire plus précisément et fermement la nouveauté et l'originalité de sa pensée $(\S 2)$. Nous nous accordons en cela avec la lecture de Walter Kaufmann qui récuse fermement en particulier l'existence d'une période «positiviste » correspondant à l'époque d'Humain, trop humain; avec les remarques de Granier (19sq.), ainsi qu'avec le propos de Wotling sur cette même question (Nietzsche et le problème de la civilisation 57 : s' «il y a bien une évolution [de la pensée de Nietzsche], c'est strictement dans le progrès de la mise en œuvre et de la maîtrise de son "nouveau langage" qu'il est possible de la déceler »). Pour cette raison, nous nous attacherons ici à confronter des textes issus d'œuvres variées de Nietzsche, afin de montrer précisément que la complexité de la réflexion nietzschéenne sur la solitude (dont la compréhension implique de réfléchir ses contradictions apparentes, que l'hypothèse initialement évoquée conduirait à ignorer et négliger) n'en exclut pas la continuité et la cohérence.

${ }^{3}$ Cf. aussi La Bruyère, Les caractères ou les mours de ce siècle, « De l'homme », 99 (1) : « Tout notre mal vient de ne pouvoir être seuls : de là le jeu, le luxe, la dissipation, le vin, les femmes, l'ignorance, la médisance, l'envie, l'oubli de soi-même et de Dieu. »

${ }^{4}$ La même idée apparaît déjà dans la Considération inactuelle III, $\S 5$ : « lorsque nous sommes seuls et silencieux, nous craignons que l'on nous souffle quelque chose à l'oreille, aussi [...] nous étourdissons-nous dans la vie de société ».

${ }^{5}$ Sur cet usage nietzschéen de la référence à Épicure, $c f$. l'étude d'Ansell-Pearson, « For Mortal Souls »141-143. Pour une étude plus générale de la relation de Nietzsche à Épicure, on se rapportera à l'étude désormais classique de Roos, « Nietzsche et Épicure ».

6 Sur ces questions, $c f$. par ex. Blondel, chap. 8; Richard Schacht, Nietzsche, chap. 2 et « Nietzsche on Philosophy, Interpretation, and Truth ».

${ }^{7}$ Cf. sur cette idée la Généalogie de la morale III, § 5, qui distingue le « désert de théâtre » qui séduit les «hommes cultivés », de celui dans lequel se trouve effectivement celui qui s'est dépris des valeurs communes. 
8 Sur ce point spécifique, $c f$. Ainsi parlait Zarathoustra, «Le retour»: «Une chose est l'abandon [Verlassenheit], une autre chose est la solitude [Einsamkeit] ».

9 L'abréviation FP désigne les fragments posthumes figurant dans l'édition des CEuvres philosophiques complètes de Nietzsche (Gallimard); elle est suivie du titre de l'œuvre ou du numéro du volume concernés, puis du numéro du fragment cité.

${ }^{10}$ Nietzsche indique aussi parfois, à cet égard, que le vivant qu'est l'homme doit lui-même être pensé comme une «collectivité inouïe d'être vivants » (FP XI 37 [4]; cf. aussi 34 [123]) : c'est un procédé de mise en abyme qui conduit cette fois à indiquer l'absence de principe ou de substance derniers.

${ }^{11}$ Sur la conception et la redéfinition nietzschéennes du corps, ainsi que sur le statut de la notion de force ou de pulsion, nous renvoyons aux études fondamentales de Müller-Lauter dans l'ouvrage Physiologie de la volonté de puissance et aux analyses de Wotling dans La pensée du sous-sol, ainsi que dans les deux articles suivants : "'L'entente de nombreuses âmes mortelles". L'analyse nietzschéenne du corps » et «"Une facilité que l'on se donne"? Le sens de la notion de pulsion chez Nietzsche », tous deux repris dans La philosophie de l'esprit libre.

12 Sur l'importance et le sens des notions de «sublimation» ou de «spiritualisation», nous renvoyons aux analyses de Wotling dans La pensée du sous-sol 96-98.

${ }^{13}$ C'est la raison pour laquelle Nietzsche peut écrire par exemple que « le courant aux cent vagues du passé nous traverse » et que nous portons en nous « des niveaux de civilisation pétrifiés » (Opinions et sentences mêlées § 223) : c'est donc également en termes historiques, dans la perspective du devenir et de l'assimilation progressive des valeurs, que la multiplicité inhérente à l'individu peut s'exprimer.

14 L'hérédité que pense Nietzsche n'est pas à comprendre dans une perspective strictement biologique, comme transmission de caractéristiques physiques: il s'agit de penser ici les conditions et la longue durée que requiert le processus de transmission et d'assimilation des valeurs qui conditionnent les manières de penser et de vivre d'une communauté ou d'un individu.

${ }^{15}$ Sur cette question spécifique, on lira par ex. Humain, trop humain I, $\S 96$ : « toute tradition se fait d'autant plus vénérable dans sa continuité que l'origine en est plus reculée, plus oubliée »; et Gai savoir \$110: «la force des connaissances ne tient pas à leur degré de vérité mais à leur ancienneté, au fait qu'elles sont incorporées, à leur caractère de condition de vie. »

${ }^{16}$ Il faut remarquer que Nietzsche n'hésite pas même à s'inscrire au sein de la communauté des «Allemands » (cf. Gai savoir §357, Par-delà bien et mal $\S 48, \S 256$ ), quelles que soient les critiques virulentes qu'il adresse à la culture allemande par ailleurs. 
${ }^{17}$ Pour cette raison, Nietzsche insiste dès la deuxième Inactuelle sur le caractère problématique de l'excès de sens historique, qui transforme cette «vertu» en "vice ». Cf. Par-delà bien et mal $\S 224$ et $\S 212$ : si le «sens historique » manque par lui-même de noblesse et de «goût», il revient à l'inverse au philosophe de savoir unifier la multiplicité que celui-ci permet d'appréhender, et de savoir surtout l'évaluer : il détermine «la valeur et le rang à partir de la multiplicité et de la diversité des choses qu'un homme pourrait supporter ».

${ }^{18}$ Sur cette idée (et plus généralement, sur l'idée de solitude comme pratique), cf. Hutter 60 : la solitude doit être pensée sur le modèle du pharmakon, c'est-à-dire comme pouvant être un remède aussi bien qu'un poison.

${ }^{19}$ On lira également sur ce point le $\S 38$ des «Incursions d'un Inactuel », dans le Crépuscule des idoles, où Nietzsche définit «[s]on concept de liberté ».

${ }^{20}$ Nietzsche thématise régulièrement cette même exigence de hiérarchisation et de sélection en mobilisant la notion de «goût » (et corrélativement celle de «dégoût ») qui permet de dire le caractère psycho-physiologique de la capacité d'évaluer et de choisir qui est celle de l'homme sain et du philosophe (cf. La philosophie à l'époque tragique des Grecs § 3, p. 223; Aurore $\S 348$; Gai savoir § 3; Par-delà bien et mal § 234; etc.).

${ }^{21}$ Ce souci concernant l'écho et la compréhension que pourraient trouver ses écrits se fait plus aigu encore à la fin de son œuvre : $c f$. par ex. Généalogie de la morale, préface $\S 8$, et Ecce homo, «Pourquoi j'écris de si bons livres » $§ 1$. Nous renvoyons sur cette question aux analyses de Wotling dans l'introduction à Nietzsche et le problème de la civilisation.

${ }^{22}$ Pour cette raison, Nietzsche insiste sur la «gaieté » qui doit surgir au sein de la communauté amicale et qui s'oppose au désespoir qui accompagne le mouvement nihiliste de dévalorisation des valeurs, l'incertitude liée à la tentative de leur recréation: «je veux les rendre plus courageux, plus résistants, plus simples, plus gais! Je veux leur enseigner ce que si peu comprennent à présent [...] : la co-réjouissance! » (Gai savoir § 338)

${ }^{23} C f$. également sur cette idée la note qui clôt le premier traité de la Généalogie, note qui requiert «un échange amical et fructueux » entre des savants issus de disciplines variées (histoire, physiologie, médecine, etc.), afin de mieux poursuivre cette étude de la «valeur des évaluations qui ont existé jusqu'à présent ».

24 Il faut bien sûr noter l'importance que Nietzsche accorde tout au long de son œuvre à la question des éducateurs, des maîtres et des disciples - le véritable disciple ne devant pas être pensé comme un simple «imitateur », mais comme celui qui est capable d'être l'adversaire de son maître et de le surpasser. $C f$. par ex. Opinions et sentences mêlées $\S 268, \S 357$, et Aurore $\S 542$.

25 On sait que Nietzsche se caractérise lui-même comme un homme «posthume » ( $c f$. par ex. Gai savoir §365; L'antéchrist, préface): par quoi il faut entendre, non seulement qu'il ne 
s'attend à être lu et compris qu'après sa mort, mais également que ses véritables ancêtres, ceux qui auraient pu le comprendre, sont eux-mêmes déjà morts — puisque telle est la signification usuelle de la formule «naître posthume ».

\section{Ouvrages cités}

Alquié, Ferdinand, Qu'est-ce que comprendre un philosophe?, Paris, La table ronde, 2005.

Ansell-Pearson, Keith, «For Mortal Souls. Philosophy and Therapeia in Nietzsche's Dawn », in C. Carlisle et J. Ganeri (dir.), Philosophy as Therapeia, Cambridge University Press, 2010, p. $137-165$.

-, «Nietzsche on Autonomy and Morality. The Challenge to Political Theory », Political Studies, vol. 39, n 2, 1991, p. 270-286.

Blondel, Éric, Nietzsche, le corps et la culture, Paris, P.U.F., 1986; rééd. Paris, L'Harmattan, 2006.

Granier, Jean, Le problème de la vérité dans la philosophie de Nietzsche, Paris, Seuil, 1966.

Hutter, Horst, « Nietzsche's Practice of Solitude », in T. E. Hart (dir.), Nietzsche, Culture, and Education, Surrey, Ashgate , 2009, p. 39-61.

Kaufmann, Walter, Nietzsche. Philosopher, Psychologist, Antichrist, Princeton University Press, 1974

Müller-Lauter, Wolfgang, Physiologie de la volonté de puissance, trad. J. Champeaux, Paris, Allia, 1998.

Nietzsche, Friedrich, Crépuscule des idoles, trad. P. Wotling, Paris, GF, 2005.

—, Le gai savoir, trad. P. Wotling, Paris, GF, 1997; rééd. 2007.

—, Généalogie de la morale, trad. P. Wotling, Paris, L.G.F., 2000.

—, Euvres philosophiques complètes, dir. G. Colli et M. Montinari, Paris, Gallimard, 18 vol., 1967-1997.

_, Par-delà bien et mal, trad. P. Wotling, Paris, GF, 2000. 
-, Sämtliche Werke. Kritische Studienausgabe, dir. G. Colli et M. Montinari, Munich/Berlin/New York, de Gruyter/D.T.V., 15 vol., 1980, rééd. 2000.

Roos, Richard, « Nietzsche et Épicure : l'idylle héroïque », in J.-F. Balaudé et P. Wotling (dir.), Lectures de Nietzsche, Paris, L.G.F., 2000, p. 283-350.

Schacht, Richard, Nietzsche, London/New York, Routledge \& Kegan Paul, 1994.

—, « Nietzsche on Philosophy, Interpretation, and Truth », Noûs, vol. 18, n 1, 1984, p. 75-85.

Tongeren, Paul J. M. van, «Politics, Friendship, and Solitude in Nietzsche (Confronting Derrida's Reading on Nietzsche in "Politics of Friendship") », South African Journal of Philosophy, vol. 19, n 3, 2000, p. 209-221.

Wotling, Patrick, Nietzsche et le problème de la civilisation, Paris, P.U.F., coll. Quadrige, 2001.

—, La pensée du sous-sol, Paris, Allia, 1999.

—, La philosophie de l'esprit libre. Introduction à Nietzsche, Paris, Flammarion, coll. Champs essais, 2008. 